\title{
Effects of Differences in Manipulation and Supporting Legs and Moving Target Speed on a Visual Tracking Test Using Center of Pressure
}

\author{
Haruka Kawabata $^{1}$, Shinichi Demura ${ }^{2}$, Masanobu Uchiyama ${ }^{3}$ \\ ${ }^{1}$ Organization of Frontier Science and Innovation, Kanazawa University, Kanazawa, Japan \\ ${ }^{2}$ Graduate School of Natural Science and Technology, Kanazawa University, Kanazawa, Japan \\ ${ }^{3}$ Akita Prefectural University, Yurihonjō, Japan \\ Email: sjskd631@ybb.ne.jp
}

Received July $31^{\text {st }}, 2013$; revised August $31^{\text {st }}, 2013$; accepted September $7^{\text {th }}, 2013$

\begin{abstract}
Copyright (C) 2013 Haruka Kawabata et al. This is an open access article distributed under the Creative Commons Attribution License, which permits unrestricted use, distribution, and reproduction in any medium, provided the original work is properly cited.
\end{abstract}

\begin{abstract}
The human limbs are paired organs, each capable of independent movement. Functional laterality is found in the upper limbs when writing letters or throwing a ball, etc. This study aimed to examine the effects of differences in manipulation leg (ML), defined as the leg used when kicking a ball and supporting leg (SL), as the contralateral leg, and moving target speed on a visual tracking test using center of pressure (COP). We included 20 healthy male students (age, $22.0 \pm 4.9$ years; height, $172.4 \pm 3.2 \mathrm{~cm}$, and weight, $66.2 \pm$ $5.0 \mathrm{~kg}$ ) without lower limb or eye disorders. During the tracking test, subjects pursued a target moving on the Y-axis by COP. We selected 0.083 and $0.050 \mathrm{~Hz}$ frequencies to examine the effect of different target speeds. An evaluation variable was defined as total errors between moving targets and COP over $30 \mathrm{~s}$. It was assumed that individuals with smaller errors would be superior during tracking tests. A significant difference was found between means for bilateral and unilateral stance (ML or SL) at both frequencies but not between ML and SL, and in all standing conditions, $0.083 \mathrm{~Hz}$ showed a smaller error than $0.050 \mathrm{~Hz}$. In conclusion, regardless of the speed of the moving target, performance of the visual tracking test was superior in bilateral than unilateral stance, and there was no difference between ML and SL. Regardless of stance, test performance reduced with faster target speed, particularly with unilateral stance (about 29\%).
\end{abstract}

Keywords: Coordination; Dynamic Balance; Agility; Limb Laterality

\section{Introduction}

The center of pressure (COP) is used as an alternative to center of mass (Hiiragi, 2008) for objective evaluation of the sway center of gravity in humans (Demura et al., 2001; Kouzaki \& Masani, 2012). COP tests for evaluation of coordination of the whole body pursuing a randomly moving target have recently been developed (Yoshida et al., 1997; Kawabata et al., 2012). These COP tests are conducted meanwhile in standing. It is necessary for subjects to integrate visual and somato sensory information with that from the vestibular apparatus and to exert appropriate leg muscles while maintaining a stable posture during the course of pursuing a randomly moving target. In short, coordination of the whole body is required to perform these tests. Coordination of balance, motor skill, dexterity, and accuracy is required and the relative contribution of these factors differs depending on the tasks involved.

Movements of the bilateral lower limbs are broadly divided into simultaneous and alternate ones, with the latter used most frequently in everyday life. Because higher stability is achieved when standing on both feet; the above tracking test is considered to be easier to accomplish in this case. Hinsie and Campbell defined laterality as the predominant use of one limb during activities such as writing, eating, watching, and hearing, etc., while Touwen defined it as one of paired organs such as hands or legs which is superior to the other in cognitive and motor skills. Because studies till today have focused mainly on the upper limbs (Nagasawa et al., 2000; Noguchi et al., 2009; Kawabata et al., 2012; Kubota et al., 2012), there is little knowledge on laterality of the lower limbs. However, Demura et al. (2010) reported that when kicking a ball, humans use one leg predominantly.

When kicking a ball, the leg used is generally termed as the manipulation leg (ML) while the contralateral leg is the supporting leg (SL) (Matsuda, 2010). When ML controls the ball, SL contributes to maintaining body stability. Hence, when performing a tracking test while standing on one leg (unilateral stance), SL may have an advantage. Therefore, it is assumed that performance of a tracking test using COP is superior in bilateral rather than unilateral stance and also when standing on SL rather than ML.

In addition, when the moving target moves faster, subjects' COP changes accordingly, leading to increased errors between moving target and COP and reduction in the accuracy of tracking the moving target. Hence, it was assumed that when the target moves faster, performance is reduced in both bilateral and unilateral stance. 
Although laterality of the upper limbs is recognized (Nagasawa et al., 2000; Kawabata et al., 2012; Noguchi et al., 2009), Matsuda et al. (2010) reported that in unilateral stance, no clear difference was found between ML and SL in a basic-level difficulty task. Because the visual tracking test used in this study demands that subjects pursue a moving target in unilateral stance without moving the feet, the level of test difficulty was considerably higher and it was assumed that the test would show a significant difference between bilateral and unilateral stance and between ML and SL.

This study aimed to determine the effects of different stances and moving target speeds on a visual tracking test.

\section{Method}

\section{Subjects}

We included 20 healthy young males (age, $22.0 \pm 4.9$ years; height, $172.4 \pm 3.2 \mathrm{~cm}$; weight, $66.2 \pm 5.0 \mathrm{~kg}$ ) without lower limb or eye disorders. The manipulation leg (ML) was defined as the one used for kicking a ball according to the survey items of Demura et al. (2010). The contralateral leg was defined as the supporting leg (SL). Before testing, the aims and procedures were explained to all subjects in detail, and written informed consent was obtained. The protocol of this study was approved by the Ethics Committee on Human Experimentation of the Faculty of Human Science, Kanazawa University.

\section{The Visual Tracking Test Using Cop}

The visual tracking test involves pursuing a moving target by COP displayed on a PC monitor. During the test, errors between moving target and COP are recorded over time. When errors were smaller, it was adjudged that subjects were capable of pursuing the target. In anatomical terms, movement of the human ankle is limited mediolaterally rather than anteroposteriorly. In the unilateral stance, displacement of the center of gravity in the mediolateral direction is more difficult than that in the anteroposterior direction. Hence, in this study, the moving target was set to move vertically on the Y-axis. An evaluation variable was the total of errors between the moving target and COP over $30 \mathrm{~s}$. When the total error was smaller, coordination was judged to be superior.

\section{Experiment Equipment}

The experimental device was a force plate prototype (Takei, Japan), comprising a force platform, A/D converter, and feedback display $(67 \mathrm{~cm}$; resolution $2560 \times 1440)$. This unit is capable of calculating the COP of vertical loads from the values of three vertical load sensors placed at the corners of an isosceles triangle on a level surface. The size of the square displayed on the monitor was $1 \mathrm{~cm}$ square and movement distance of the target was the same as that displayed on the monitor (Figures 1 and 2).

\section{Experimental Condition}

The sampling time and frequency were $30 \mathrm{~s}$ and $50 \mathrm{~Hz}$, respectively. Yoshida et al. conducted a similar test with bilateral stance over $60 \mathrm{~s}$, but we used a shorter time to avoid the likelihood of subjects' fatigue of lower limbs due to being performed by bilateral stance. The monitor for visual feedback was placed at a distance of $1.5 \mathrm{~m}$ from subjects and at eye level.

\section{Test and Test Procedure}

Subjects stood holding their arms down with feet $5 \mathrm{~cm}$ apart (bilateral stance) or with one foot on the center of the platform (unilateral stance) (Figure 3). Measurement was started following a sign given by the tester. After one practice trial, subjects performed 5 further trials with breaksofl min between trials. To avoid the effect of order, measurement of unilateral stance was performed at random. The target moved within a range of $\pm 3.0 \mathrm{~cm}$ around the center on the feedback monitor. It moved along the sinusoidal waveform with amplitude of $3.0 \mathrm{~cm}$ on the Y-axis at a speed of either $0.083 \mathrm{~Hz}(1.0 \mathrm{~cm} / \mathrm{s})$ or 0.050 $\mathrm{Hz}(6.0 \mathrm{~cm} / \mathrm{s})$. Subjects' COP was set to automatically display the origin on the screen at the start of the test.

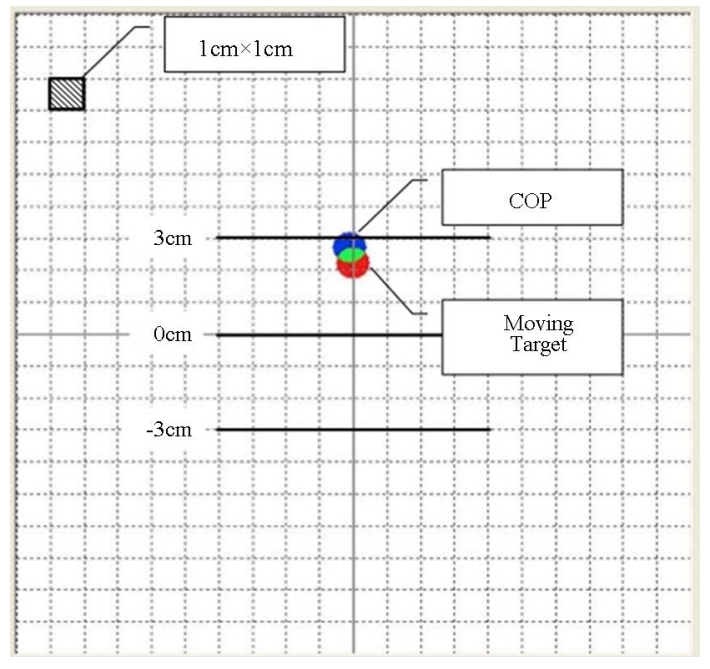

Figure1.

View of the monitor screen during testing.

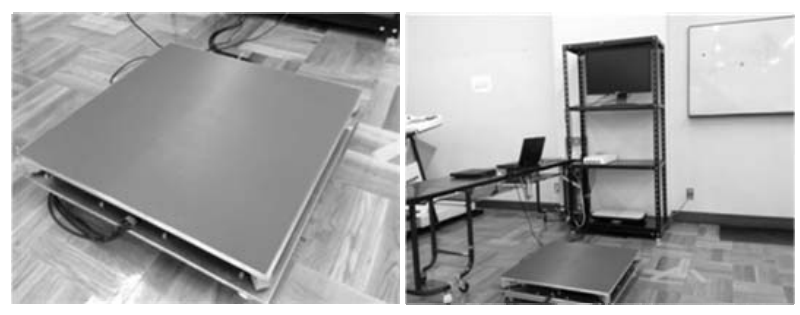

(a)

(b)

Figure2.

Experimental equipment.

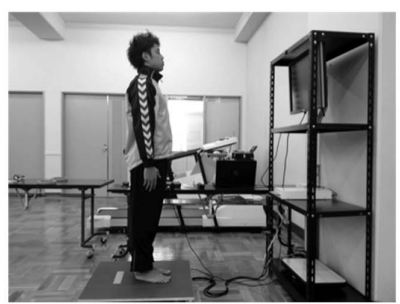

(a)

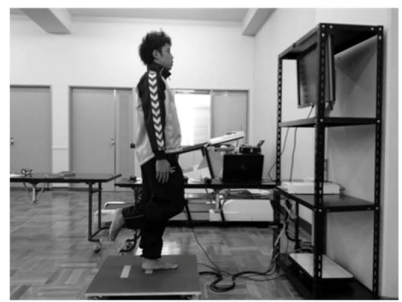

(b)
Figure 3.

Measurement scenery ((a) Both legs, (b) One leg). 


\section{Statistical Analysis}

SPSS $10.4 \mathrm{~J}$ software package (IBM, USA) was used for data analysis. The representative value for all conditions tested was the mean of three trials (maximum and minimum values were excluded). Two-way analysis of variance (ANOVA) with correspondence in only one factor was used to test differences among means. When significance was found in the main factor or interaction, a multiple comparison test using Tukey's HSD method was performed. The level of significance was set a priori at 0.05 in this study.

\section{Results}

Table 1 shows the mean and standard deviation for bilateral leg (BL), ML, and SL at both 0.083 and $0.050 \mathrm{~Hz}$. A significant difference was found in the factors "main effect of moving target speed" and "stance". Results of multiple comparison showed that the mean at both 0.083 and $0.050 \mathrm{~Hz}$ was lower in BL (772.8) than in both ML (885.8) and SL (906.1), but no significant difference was found between means of ML and SL. In addition, the mean at $0.050 \mathrm{~Hz}$ was significantly lower than that at $0.083 \mathrm{~Hz}$ for BL, ML, and SL. Effect of size (ES) at $0.083 \mathrm{~Hz}$ was 1.26 between BL and MS and 1.50 between BL and SL. ES at $0.050 \mathrm{~Hz}$ was 2.32 between BL and ML and 2.83 between BL and SL.

\section{Discussion}

\section{Different Stance and Speed of Moving Target Affect the Visual Tracking Test When Using Cop}

Movement of the ankle joint plays an important role during physical activities and also markedly affects posture control. If ankle motion is not smooth, maintaining a stable posture against perturbation in addition to walking and running becomes difficult. In this study, to evaluate postural control ability before and after direction, a tracking test using COP was conducted under conditions of different target speed ( 0.050 and $0.083 \mathrm{~Hz}$ ) and different stance (BL, SL, and ML).

Bilateral stance is generally more stable than unilateral because of larger base of support. In contrast, ML is generally used when kicking a ball or treading. When ML is used during certain movements, SL maintains a stable posture to allow ML to function normally. In short, the two legs perform different tasks at the same time. The present findings show that performance in unilateral stance is significantly lower than that in bilateral; therefore, bilateral stance and higher stability are considered to positively influenced performance of the tracking test.

Touwen defined laterality as one of paired organs such as hands or legs is superior to the other in the performance of cog- nitive and motor skills. Nagasawa et al. (2000), Kawabata et al. (2012), and Noguchi et al. (2009) reported that laterality exists in the upper limbs and that the dominant hand was superior in the purdue pegboard, the pursuit rotor, and the coordinated force exertion tests. Coren investigated the dominant leg in a study of 3307 males and females (age, 17 - 35 years) and reported that $83.9 \%$ of males and $88.9 \%$ of females tended to have the right leg as the dominant lower limb. In the present study, the ML for all subjects was the right leg.

In a visual tracking test, it was assumed that when standing on ML it would be easy to pursue the moving target to obtain good results. However, a nonsignificant difference was found between the means of SL and BL. Either side can be used predominantly in the upper limbs but the lower limbs are used equally during activities such as walking, running, etc. In short, because neither leg is used predominantly, it is inferred that any difference between ML and SL is as marked as that between dominant and nondominant upper limbs.

\section{Effects of Speed of a Moving Target on Visual Tracking Test Using Cop}

The tracking test using a moving target is similar to balance tests such as the cross test (Tsukimura \& Ikeda, 1982) and the functional reach test (Dancan et al., 1990) in terms of displacement of the COP with legs stationary as the support base. According to Hase (2006), the center of gravity is displaced forward when the soleus muscle is activated and backward when the anterior tibial and quadriceps muscles are activated. In short, the lower limb muscle group (e.g., quadriceps femoris muscle, hamstrings, tibial is anterior muscle, triceps surae muscle) is most involved in maintaining standing posture. In this tracking test, subjects were required to operate the feed-forward control to displace the COP either forward or backward according to movements of the target and to move the center of gravity quickly when the speed of the moving target increased. It was assumed that the burden on the leg muscle group increases because of the above effect. We inferred that any factors increasing the speed of the moving target increase the difficulty of the test.

Kawabata et al. (2012) conducted a tracking test on subjects in bilateral stance using a target moving at an average speed of $0.083 \mathrm{~Hz}$. However, the present study used two different test speeds to examine the effect of these to include testing in unilateral stance.

Tracking test scores were less for ML and SL at $0.083 \mathrm{~Hz}$ than at $0.050 \mathrm{~Hz}$, with the error at $0.083 \mathrm{Hzbeing}$ greater than that at $0.050 \mathrm{~Hz}$ by approximately $4 \%$ and $29 \%$ for bilateral and unilateral stance, respectively. This implies that it was difficult for subjects to pursue the moving target when its speed increased.

The visual tracking test used in this study can be useful in

Table 1.

Mean, standard deviation, and results of ANOVA in visual tracking test using COP.

\begin{tabular}{ccccccccccc}
\hline & \multicolumn{2}{c}{ BL } & \multicolumn{2}{c}{ ML } & \multicolumn{2}{c}{ SL } & \multicolumn{3}{c}{ Two-way ANOVA } & Post-hoc of HSD \\
\hline & Mean & SD & Mean & SD & Mean & SD & F & $p$ & Partial $\eta^{2}$ & \\
$0.083 \mathrm{~Hz}$ & 772.8 & 160.2 & 885.8 & 192.8 & 906.1 & 227.7 & $18.77^{*}$ & 0.00 & 0.33 & All conditions: $0.083 \mathrm{~Hz}>0.05 \mathrm{~Hz}$ \\
$0.05 \mathrm{~Hz}$ & 541.3 & 92.7 & 686.2 & 140.6 & 704.8 & 139.6 & $50.80^{*}$ & 0.00 & 0.57 & $0.083 \mathrm{~Hz}: \mathrm{BL}<\mathrm{ML}(0.64), \mathrm{SL}(0.68)$ \\
& & & & & & & 0.63 & 0.54 & 0.02 & $0.05 \mathrm{~Hz}: \mathrm{BL}<\operatorname{ML}(1.21), \mathrm{SL}(0.49)$ \\
\hline
\end{tabular}

Note: ${ }^{*} p<0.05$, F1: Speed of moving target, F2: Standing posture, F3: Interaction, (): effect size BL: both legs, ML: manipulation leg, SL: supporting leg. 
evaluating the ability to coordinate COP with a moving target using visual feedback. It was determined that test performance was more stable in bilateral than in unilateral stance, and laterality was not found in the lower limbs. Although the coordination ability of the whole body is very important for skillful competitive sports, the development of a simple and practical evaluation test has not received much attention till date. In addition, during certain sports events (e.g., soccer), coordination in unilateral stance is more important than that in bilateral stance (e.g., ski-jumping). From the findings of this study, in case of athletes requiring high levels of skill, a coordination test in unilateral stance involving a high level of difficulty provides useful information, and distinction between dominant and nondominant leg may be unnecessary.

\section{Conclusion}

In conclusion, regardless of the speed $(0.083 / 0.050 \mathrm{~Hz})$ of the moving target, performance in the tracking test was superior in bilateral stance than in unilateral stance, but no differences between ML and SL were noted. Regardless of the stance, performance declines when the speed of the moving target increases.

\section{REFERENCES}

Cohen, J. (1988). Statistical power analysis for the behavioral sciences (2nd ed.). Hillsdale, NJ: Lawrence Erlbaum.

Coren, S. (1993). The Lateral Preference Inventory for measurement of handedness, footedness, eyedness, and earedness: Norms for young adults. Bulletin of the Psychonomic Society, 31, 1-3. http://dx.doi.org/10.3758/BF03334122

Demura, S., Yamaji, S., Noda, M., Kitabayashi, T., \& Nagasawa, Y. (2001). Examination of parameters evaluating the center of foot pressure in static standing posture from the viewpoints of trial-to-trial reliability and interrelationships among parameters. Equilibrium Research, 60, 44-55. http://dx.doi.org/10.3757/jser.60.44

Demura, S., Sato, S., \& Sugiura, H. (2010). Lower limb laterality characteristics based on the relationship between activities and individual laterality. Gazzetta Medica Italiana, 169, 169-181.

Dolcos, F., Rice, H. J., \& Cabeza, R. (2002). Hemispheric asymmetry and aging: Right hemisphere decline or asymmetry reduction. Neuroscience \& Biobehavioral Reviews, 26, 819-825.

http://dx.doi.org/10.1016/S0149-7634(02)00068-4
Duncan, P. W., Weiner, D. K., Chandler, J., \& Studenski, S. (1990). Functional reach: A new clinical measure of balance. The Journals of Gerontology, 45, 192-197.

http://dx.doi.org/10.1093/geronj/45.6.M192

Geshwind, N., \& Behan, P. (1982). Left-handedness: Association with immune disease, migraine, and developmental learning disorder. Proceedings of the National Academy of Sciences, 79, 5097-5100. http://dx.doi.org/10.1073/pnas.79.16.5097

Hase, K. (2006). Postural control for quiet standing. Japanese Journal of Rehabilitation Medicine, 43, 542-553.

Hiiragi, Y. (2008). Measuring and comparing the base of support and movable range of center of gravity. Rigakuryohokagaku, 23, 229234.

Kawabata, H., Demura, S., Kitabayashi, T., \& Sato, S. (2012). Gender and the laterality of various coordination tests. Gazzetta Medica Italiana, 173, 135-141.

Kawabata, H., \& Demura, S. (2012). Reliability and sex difference in a coordination test of a tracking moving target with the center of foot pressure. Advances in Physical Education, 2, 77-81. http://dx.doi.org/10.4236/ape.2012.23014

Kozaki, M., \& Masani, K. (2011). Postural sway during quiet standing is related to physiological tremor and muscle volume in young and elderly adults. Gait Posture, 35, 11-17. http://dx.doi.org/10.1016/j.gaitpost.2011.03.028

Kubota, H., Demura, S., \& Kawabata, H. (2012). Laterality and agelevel differences between young women and elderly women in controlled force exertion (CFE). Archives of Gerontology and Geriatrics, 54, 68-72. http://dx.doi.org/10.1016/j.archger.2011.06.027

Matauda, S., Demura, S., \& Demura, T. (2010). Static one-legged balance in soccer players during use of a lifted leg. Perceptual \& Motor Skills, 111, 167-177. http://dx.doi.org/10.2466/05.23.26.27.PMS.111.4.167-177

Noguchi, T., Demura, S., \& Aoki, H. (2009). Superiority of the dominant and nondominant hands in static strength and controlled force exertion. Perceptual \& Motor Skills, 109, 339-46. http://dx.doi.org/10.2466/pms.109.2.339-346

Touwen, B. C. L. (1972). Laterality and dominance. Developmental Medicine \& Child Neurology, 14, 747-755. http://dx.doi.org/10.1111/j.1469-8749.1972.tb03318.x

Tsukimura, T., \& Ikeda, T. (1982). Clinical study on safety area of standing the cross test for cerebral palsy. Journal of Rehabilitation Medicine, 19, 25-32. http://dx.doi.org/10.2490/jjrm1963.19.25

Yoshida, T., Oda, M., Osafune, H., Miyaji, M., \& Yamamoto, M. (1997). The evaluation of tracking ability by the body tracking (BTT). Equilibrium Research, 56, 39-44. http://dx.doi.org/10.3757/jser.56.39 